\title{
Der Nutzen von Produktbilanzen für den Verbraucher
}

Kauf- und Nutzungsentscheidungen sind Elemente des alltäglichen Lebens, die nicht selten gewohnheitsmäßig, willkürlich oder unwillkürlich getroffen werden, ohne dabei in das Bewußtsein der VerbraucherInnen vorzudringen. Bei Kaufentscheidungen, die bewußt getroffen werden, sind die VerbraucherInnen einer Flut von oft widersprüchlichen Informationen und Bedürfnissen ausgesetzt. Dabei ist zu beachten, daß der von den Verbrauchern erwartete Nutzen aus den Produkten sich aus vielen Komponenten zusammensetzt: dem Gebrauchsnutzen, dem Erlebnisnutzen, dem Image- oder dem Prestigenutzen bspw.(1) Damit entziehen sich Kauf- und Nutzungsentscheidungen je nach Produkt mehr oder weniger rationalen, von außen nachvollziehbaren Gründen. Informationen über (ökologische) Produkteigenschaften, wie Produktbilanzen sie bereitstellen, können daher nur »nützen «, wenn Verbraucher zwischen zwei oder mehreren Produkten mit gleichem Gebrauchs-, Erlebnis- oder Ausstattungsnutzen wählen können.

Konzepte und Methoden, VerbraucherInnen verdichtete herstellerneutrale Informationen über Produkte und ihre Wirkungen bereitzustellen, existieren in verschiedenster Form: Tests der Stiftung Warentest und der Zeitschrift Öko-Test sowie die Kennzeichnung z. B. mit dem Blauen Engel. Diese Instrumente sollen die VerbraucherInnen in vereinfachter Form über eine Fülle von Produktmerkmalen informieren und damit in die Lage versetzen, gezieltere Kauf- und Nutzungsentscheidungen treffen zu können. Solche Produktbewertungen sind jedoch mit Mängeln versehen:

1. Die Produkte werden nur in Bezug auf wenige Bereiche ihres Lebens, das von der Rohstoffgewinnung bis zur Entsorgung reicht, bewertet.

2. Es werden nur einige wenige ökologische Auswirkungen berücksichtigt.

Um umfassendere Bewertungen von Umweltauswirkungen vornehmen zu können, wurden etliche weitergehende Instrumente entwickelt: Ökobilanz, Produktlinienanalyse, Produkt-Umweltverträglichkeitsprüfung, Prozeßkettenanalyse, Vergleichende Umweltbilanz, Ökologische Systemanalyse, Life-Cycle-Analysis. Diese Instrumente unterscheiden sich hinsichtlich ihrer Methodik, ihrer Zeithorizonte, ihrer Ziele und dem Bewertungsanspruch, wobei die Produktlinienanalyse neben ökologischen auch gesellschaftliche und wirtschaftliche Aspekte einbezieht (2). Produkt-Öko-Bilanzen werden im Bereich der Verbraucherberatung zunehmend häufiger angewendet. Gerade bei den Produktbilanzen, mit denen oftmals Produkte verschiedener Hersteller verglichen werden, divergieren Anspruch sowie Neutralität der Untersuchungen in starkem Maße.

\section{Was bieten Produktbilanzen?}

Bei der Produktbilanzierung werden die umweltbezogenen Auswirkungen eines oder mehrerer Produkte untersucht. In Parameterstudien werden bspw. die Produkte verschiedener Hersteller untersucht oder verschiedene Produktalternativen mit unterschiedlichen Techniken, z. B. Haushaltskühlgeräte mit Verdampfer- bzw. Ammoniaktechnik, Händetrocknen mit Papier- handtüchern oder Gebläse, verglichen. Produktbilanzen sind nicht zu verwechseln mit unternehmensbezogenen Öko-Bilanzen: hier werden die Umweltauswirkungen eines Unternehmens untersucht: die Herstellung der Produkte, Rohstoffgewinnung, Energie- und Materialeinsatz, Transport, bis zu Verbrauch und Entsorgung. Wenn Betriebe solche Öko-Bilanzen über Jahre fortschreiben, können sie auch in ein umfassenderes Konzept wie das Öko-Controlling übergehen, in das z.B. auch organisatorische Aspekte einbezogen werden. Öko-Bilanzen können auch als Grundlage für ein Prüfverfahren wie das Öko-Auditing dienen.

Produktbilanzen unterscheiden sich hinsichtlich ihrer Interessengebundenheit, der Ausführlichkeit ihres Untersuchungsrahmens, der Aktualität und Detailgenauigkeit der Daten sowie der Transparenz ihrer Bewertung. Der Nutzen von Produktbilanzen für die VerbraucherInnen hängt damit im wesentlichen von der Glaubwürdigkeit, der Transparenz, der Aussagekraft und der Verständlichkeit der Ergebnisse ab.

\section{Welche Probleme ergeben sich} bei der Produktbilanzierung?

Zur Erstellung von Produktbilanzen werden eine Fülle von hochkomplexen Daten verarbeitet und einer Bewertung unterzogen. Diese Komplexität muß verbrauchergerecht reduziert werden. Bei einer solchen Reduktion besteht immer eine Gratwanderung zwischen der Genauigkeit und Verständlichkeit von komplexen Informationen. Dabei gilt, daß die Abhängigkeit von Expertenwissen steigt, je komplexer die Informationen sind

Für Produktbilanzen hat sich noch kein einheitlicher Methodenstandard herausgebildet, obwohl inzwischen Bemühungen zur Vereinheitlichung erkennbar sind. Genausowenig existieren klare Anforderungen an Daten oder Begrifflichkeiten. So entsteht die Situation, daß Produktbilanzierungen bei gleichem Untersuchungsgegenstand zu unterschiedlichen Ergebnissen kommen können. Dies ist auf folgende Probleme zurückzuführen:

a) Festlegung des Untersuchungsrahmens,

b) Qualität der Daten, c) Beschreibung des Produktsystems,

d) Beachtung der funktionalen Äquivalenz,

e) Zusammenfassung und Bewertung der Ergebnisse.

a) Festlegung des Untersuchungsrahmens $\mathrm{Zu}$ einer Produktbilanz gehört die Betrachtung des gesamten Produktlebenszyklus (von der Wiege bis zur Bahre) sowie die Berücksichtigung der ökologischen Auswirkungen auf jeder Lebenszyklusstufe. Diese Anforderung findet in kaum einer Produktbilanz in ihrer gesamten Bandbreite Eingang. Transportwege z. B. für die einzelnen Materialien, die zur Herstellung eines Produktes notwendig sind, finden meist keinen Niederschlag. Tropenhölzer werden unter der Rubrik »nachwachsend « verbucht, unabhängig davon, ob sie durch Abholzung des nicht wieder aufforstbaren Regenwaldes oder durch Plantagenbau gewonnen werden.

Die ökologischen Auswirkungen werden meist über die Auflistung der Emissionen in Bezug auf Ressourcenverbrauch, Luft- und Wasseremissionen, Abfallaufkommen und Flächenversiegelung erfaßt. Das Kriterium Abfall bspw. wird häufig mit dem Indikator »Gewicht« beschrieben. Damit werden Aspekte wie Toxizität oder Volumen vernachlässigt. Die komplette Erfassung aller Umweltauswirkungen stellt ein höchst umfangreiches Vorhaben dar.

\section{b) Qualität der Daten}

Die Aussagekraft einer Produktbilanz hängt von der Art, Qualität und Repräsentativität der verwendeten Daten ab. Bei der Qualität der Daten existieren zwei Problembereiche:

1. Viele Umweltauswirkungen können nicht nur einem Produkt zugeordnet werden. Alle Daten sind raum- und zeitabhängig und dem jeweiligen Forschungsstand und der Interpretation der Ergebnisse zuzuordnen. Da oftmals konkrete Informationen fehlen, müssen viele Daten geschätzt werden.

2. Viele erhobene Daten unterliegen der Geheimhaltung durch den Auftraggeber und können daher nicht genutzt werden. Als Problem ist der Umgang mit dem Datenmangel zu sehen. Viele Produktbilanzen weisen nicht explizit aus, welche Daten fehlen bzw. unter welchen Bedingungen sie geschätzt wurden. Als Metakriterien müssen daher Transparenz, Reproduzierbarkeit, Fairness und Offenlegung der Datenquellen gelten.

\section{c) Beschreibung des Produktsystems}

Bei der Beschreibung des Produktsystems (Beschreibung des Produktionsvorgangs und welche Einflußfaktoren wirksam werden) gibt es eine ganze Reihe von Spielräumen:

- Technologischer Entwicklungsstand: Geht man z. B. bei der Produktbilanzierung eines Zellstoffprodukts davon aus, daß eine Produktionsanlage mit Kreislaufwasserführung zum Standard gehört, ergeben sich 
andere Relationen, als wenn das nicht als selbstverständlich vorausgesetzt wird.

- Kuppelproduktion: Mit welcher Gewichtung der Material- und Energieeinsatz des Ausgangsstoffes in die einzelne Produktbilanz fließt, ist Ermessenssache.

- Energiesystem: Die Energie, die für die Herstellungsprozesse oder Verbrauchsphasen benötigt wird, kann aus verschiedenen Energieträgern stammen (In Schweden z. B. hauptsächlich Wasserkraft, bei uns in Deutschland hauptsächlich Kohle. Einzelne Firmen setzen schon eigene Energiesysteme ein, z. B. Solaranlagen oder Windräder).

- Transportsystem: Zwischen den einzelnen Lebensstufen eines Produktes finden verschiedene Transportvorgänge statt. Mit welchen Verkehrsmitteln in welcher Auslastung transportiert wurde, wird oftmals nicht explizit berücksichtigt.

- Abfallverwertung: Die Frage, ob ein Produkt nach dem Gebrauch wiederverwertet, recycled oder entsorgt wird und unter welchem Aufwand (Energie) bzw. welchen Umweltauswirkungen, kann oftmals auch wegen zu großer Zeithorizonte nicht abschließend beantwortet werden.

\section{d) Beachtung der funktionalen Äquivalenz}

Die meisten Produktbilanzen vergleichen mehrere Produkte oder Konsumvorgänge miteinander, um deren Umweltverträglichkeit gegenüberzustellen. Um einen fairen Vergleich durchzuführen, ist es notwendig, daß die Vergleichsprodukte funktional äquivalent sind, $d$. $h$. die gleiche Leistungsfähigkeit besitzen. Kriterien für Produkteigenschaften und deren Gleichheit werden objektiv schwer zu finden sein. Es ist jedoch möglich und notwendig, die für gleich befundenen Eigenschaften im Bilanzierungsbericht herauszustellen.

\section{e) Zusammenfassung und Bewertung} der Ergebnisse

Das Zusammenfassen von Daten zu einer überschaubaren Menge bedeutet immer, daß diese gewichtet und gewertet werden. Einer Bewertung liegen grundsätzlich Werturteile zugrunde über die relative Wichtigkeit von Umweltauswirkungen und die bei deren Erfasssung relevanten Umweltkriterien. Neue wissenschaftli-

\section{IÖW-'Adressen}

\section{IÖW/VöW-Geschäftsstelle}

Giesebrechtstraße 13, 10629 Berlin

Tel. (030) 8826094 , Telefax 8825439

IÖW-Regionalbüro Nordrhein-Westfalen Völklinger Straße 9, 42285 Wuppertal Tel. (02 02) 80530, Telefax 83402

IÖW-Regionalbüro Schleswig-Holstein Waitzstraße 11, 24105 Kiel

Tel. (0431) 566362, Telefax 578336 che Erkenntnisse können darüber hinaus die Aussage einer Produktbilanz völlig in Frage stellen und die Relevanz eines Umweltkriteriums kann sich im Zeitablauf verändern.

Bei der Zusammenfassung der Daten geht es um einen Konflikt zwischen Operationalität und Komplexität. Fällt die Gewichtung zugunsten der Operationalität, sind die Metakriterien: Transparenz, Reproduzierbarkeit, Fairness und Offenlegung der Datenquellen nicht gewährleistet; wird zugunsten der Komplexität entschieden, ist die Aussagekraft stark eingeschränkt (3).

\section{Welchen Nutzen haben Produktbilanzen für den Verbraucher?}

Produktbilanzen sind, verglichen mit anderen Instrumenten, sehr umfassende Mittel, VerbraucherInnen bei Kauf- und Nutzungsentscheidungen, verdichtete Informationen zu liefern. Unter der Voraussetzung, daß die systemimanenten Probleme vòn Produktbilanzen gelöst werden können, indem:

- eine einheitliche Untersuchungs- und Bewertungsmethodik aufgestellt werden kann,

- ihre Daten an die dynamische Entwicklung von erkenntnistheoretischen und datenrelevanten Forschungsergebnissen angepasst werden,

- die Ergebnisse verständlich dargestellt werden,

können Produktbilanzen eine umfassende und informative Entscheidungshilfe sein.

Produktbilanzen sind immer in ihrem Kontext zu sehen. Ihre Aussagen beziehen sich nur auf die definierten Systemgrenzen. Daher sollte bei jeder Produktbilanz zunächst die Zielsetzung, die Methodik und die Äquivalenzeigenschaften deutlich herausgestellt werden. Erforderlich wäre als Grundlage ein einheitlicher Methodenund Bewertungsstandard, der die Differenzen zwischen den Ergebnissen gleicher Untersuchungsgegenstände so gering wie möglich hält. Ebenso notwendig ist die zeitliche Anpassung der Daten an den Stand der Forschung und damit mögliche Änderungen der Ergebnisse. Auf diese Weise werden Produktbilanzen nicht nur aktualisiert, sondern mögliche Reaktionen auch dokumentiert und damit gefördert.

IÖW-Regionalbüro Baden-Württemberg

Bergheimerstraße 97, 69115 Heidelberg Tel. (06221) 167954, Telefax 27060

IÖW-Projektbüro Hannover

Hausmannstraße 9, 30159 Hannover

Tel. (05 11) 1640344, Telefax 1640391

IÖW-Österreich, Geschäftsstelle

Rechte Wienzeile 19/5, A-1043 Wien

Tel. (0043-222) 5872189
Zur Verständlichkeit und Nachvollziehbarkeit der Ergebnisse könnten Produktbilanzen in drei parallelen Stufen dargestellt werden:

1. Stufe: Kurze Darstellung des Untersuchungsgegenstandes und der zusammenfassenden Bewertung in einfachen Worten und gut sichtbar (z. B. Kasten),

2. Stufe: Darlegung der Untersuchungsziele, Methodik, Äquivalenzeigenschaften, Zusammenfassung der Ergebnisse der einzelnen Untersuchungsgebiete, zusätzlich zur ersten Stufe, 3. Stufe: Dokumentation des gesamten Prozesses und der Ergebnisse der Produktbilanzierung, zusätzlich zur zweiten Stufe.

Die verschiedenen Stufen könnten auf unterschiedliche Medien verteilt werden. $\mathrm{Zu}$ wünschen ist, daß mindestens die erste Stufe mit Hinweis auf die Bezugsadresse für die weiteren Stufen auf oder an den Produkten selbst plaziert ist. Der weitaus größte Teil der Kaufentscheidungen wird im Geschäft getroffen und, wie eingangs erwähnt, oftmals aus Gewohnheit oder willkürlich. Dabei sind am Produkt oder innerhalb des Geschäftes angebrachte Produktinformationen in Form von Produktbilanzen möglicherweise ein Anlaß für die VerbraucherInnen, gezielter zu entscheiden. Das größte Problem dürfte die mangelnde Bereitschaft der Hersteller sein, auch negative Eigenschaften ihres Produktes offenzulegen. Selbst für Hersteller, deren Produkt positiv abschneidet, bleibt zu klären, ob der unmittelbare Vergleich mit weiteren Produkten der Produktbilanz auf oder an dem eigenen Produkt wettbewerbsrechtlich statthaft ist. Insofern muß realistischerweise davon ausgegangen werden, daß ein neutraler Verteiler wie Zeitschriften, Verbraucherzentralen, Beratungsstellen in Frage kommt. Hier wäre allerdings der Nachteil, daß wiederum nur interessierte und problembewußte VerbraucherInnen, die selbst aktiv werden, von den Produktbilanzergebnissen profitieren könnten.

Des weiteren darf nicht übersehen werden, daß Produktbilanzen keine Entscheidungshilfe bezüglich des Bedarfsnutzens bieten, sondern meist nur die Alternative zwischen größeren und kleineren Übeln, da sie von der Methodik her dem Prinzip des Verursachers von schädigenden Einflüssen folgen. Alternativen, die den ermittelten Umweltschäden eventuell vorbeugen, werden nicht benannt. Eine weiterentwickelte Produktbilanz müßte jedoch aufzeigen, welche Alternativen neben den Untersuchungsgegenständen existieren. Die Entscheidung, ob ein Produkt überhaupt benötigt wird, wird immer bei den Verbrauchern selbst liegen. Den VerbraucherInnen, die ökologisch ganz sicher gehen wollen, bleibt nur der Konsumverzicht.

\section{Cornelia Weskamp, Wuppertal}

(1) Für weitere Fehlentwickungen des Konsums vgl U. Beier: Der fehlgeleitete Konsum, Frankfurt 1993 (2) Stölting/Rubik: Übersicht über ökologische Produktbilanzen, IÖW Heidelberg 1992

(3) StöIting/Rubik 1992 
(c) 20I0 Authors; licensee IÖW and oekom verlag. This is an article distributed under the terms of the Creative Commons Attribution Non-Commercial No Derivates License (http://creativecommons.org/licenses/by-nc-nd/3.o/), which permits unrestricted use, distribution, and reproduction in any medium, provided the original work is properly cited. 